\title{
Treatment of a patient with Kawasaki disease associated with selective IgA deficiency by continuous infusion of cyclosporine A without intravenous immunoglobulin
}

\author{
Tatsuya Anzai, Takaomi Minami, Tomoyuki Sato, Sadahiro Furui, Takanori Yamagata \\ Department of Pediatrics, Jichi Medical University, Yakushiji, Japan. \\ E-mail: tminami@jichi.ac.jp \\ Received: 10th March 2016, Revised: 6th May 2016, Accepted: 12th July 2016
}

\begin{abstract}
SUMMARY: Anzai T, Minami T, Sato T, Furui S, Yamagata T. Treatment of a patient with Kawasaki disease associated with selective IgA deficiency by continuous infusion of cyclosporine A without intravenous immunoglobulin. Turk J Pediatr 2016; 58: 666-668.

Intravenous immunoglobulin therapy is standard for Kawasaki disease (KD) treatment; however, anaphylactic reactions to immunoglobulins are a risk in KD patients with selective IgA deficiency (sIgAD). The therapy for KD associated with sIgAD has not been established. The IgA immune response is believed to play an important role in KD vasculitis. We report the case of a 5-year-old boy with KD and sIgAD treated with intravenous cyclosporine A (CsA, $3.0 \mathrm{mg} / \mathrm{kg} /$ day) instead of intravenous immunoglobulin (IVIG). The fever and inflammation immediately resolved without a coronary artery lesion. In KD patients with $\operatorname{SIgAD}$, we believe that an IgA immune response is lacking, which is the reason for milder KD symptoms than in those without sIgAD. This case report aids in clarifying the role of IgA antibodies in KD and provides evidence that CsA is a potential candidate for first-line therapy for patients with KD with contraindications to IVIG.
\end{abstract}

Key words: cyclosporine A, Kawasaki disease, selective IgA deficiency, coronary artery lesion.

Immunoglobulin (Ig) A deficiency is defined as a decreased or absent level of serum IgA, with normal serum levels of IgG and IgM in patients aged more than 4 years. Usually, a serum IgA level $<7.0 \mathrm{mg} / \mathrm{dl}$ is considered as selective $\operatorname{IgA}$ deficiency $(\operatorname{sg} A D)^{1}$. The incidence of sIgAD ranges from 1:14,840 to $1: 18,500$ in Japan ${ }^{2}$. IgA-deficient patients may produce anti-IgA antibodies, and have a potential risk of anaphylactic reactions to the transfusion of any blood products which contain trace amounts of $\operatorname{IgA}$, such as red blood cells or immunoglobulins ${ }^{1}$. Cyclosporine A (CsA) is used for patients with intravenous immunoglobulin (IVIG)-resistant Kawasaki disease $(\mathrm{KD})^{3-5}$. There have been no reports of patients with KD initially treated with CsA without IVIG.

The etiology of KD is still unknown. IgA plasma cells infiltrate the vascular system in acute and subacute phases of KD, and it should be noted that IgA immune response assumes an important role in the pathophysiology of $\mathrm{KD}^{6,7}$. However, how the IgA immune response works in acute or subacute phase KD with SIgAD is unclear. We report the case of a patient with $\mathrm{KD}$ associated with sIgAD. He was treated with continuous infusion of CsA (CICsA) instead of IVIG and was cured immediately without coronary artery lesion (CAL).

\section{Case Report}

A 5-year-old boy, presented with persistent fever lasting 6 days, cervical lymphadenopathy, red bulbar conjunctival injection, cracked lips and tongue, and brawny edema of the hands. There was no induration at the BCG inoculation site. He met the criteria for KD with a score of $5 / 6$, and was diagnosed with sIgAD based on his serum IgA level $(<5.0 \mathrm{mg} / \mathrm{dl})$. He had had respiratory tract infections every two months for 3-5 years. He also had cellulitis after dental 


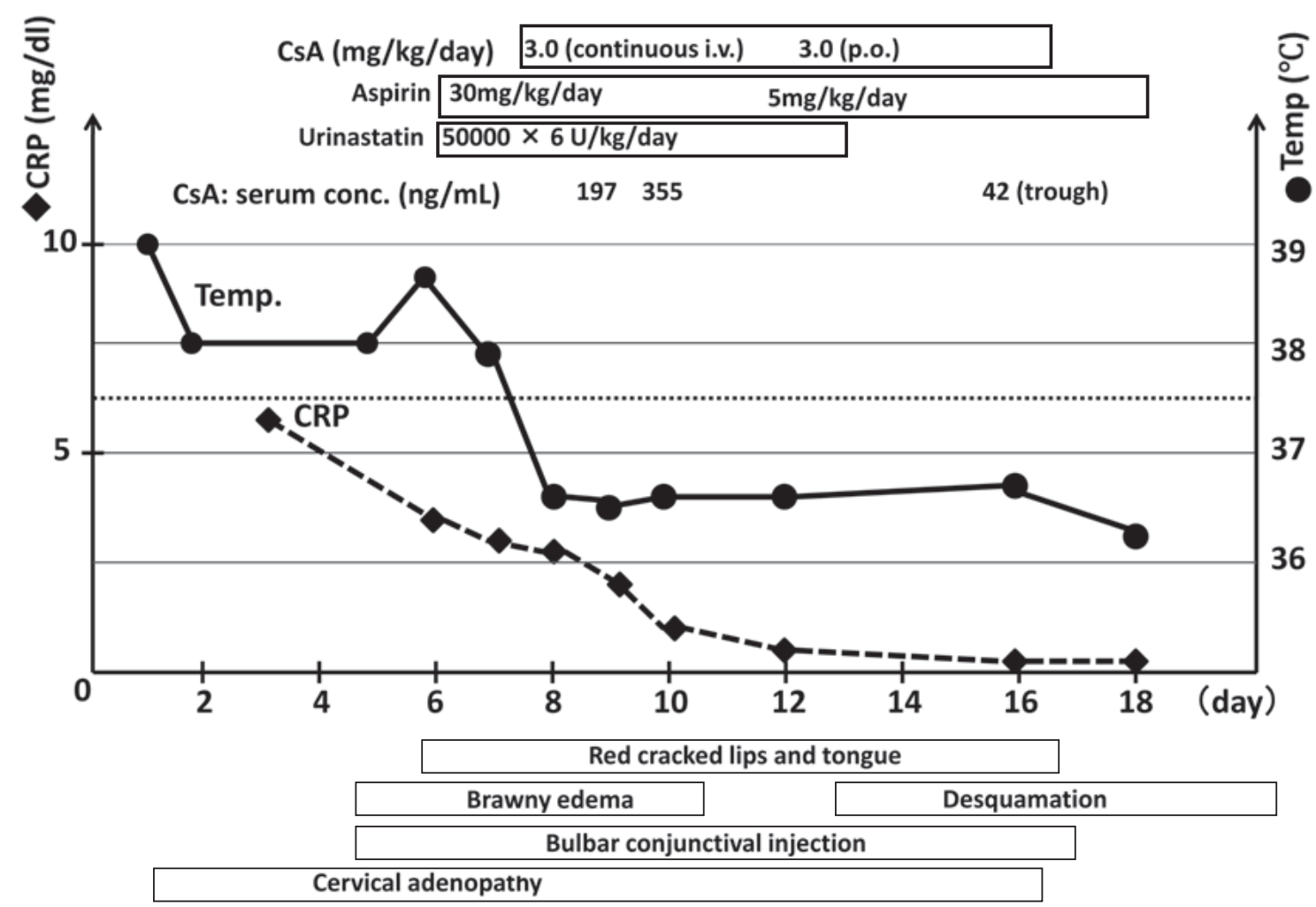

Fig. 1. Clinical course of the patient. CSA: cyclosporin A.

therapy when he was 3 years old. He had no history of blood product exposure, including IVIG. Serum IgA concentrations of his parents and the younger brother were normal.

Physical examination results on admission were as follows: height $110.2 \mathrm{~cm}(+0.4$ standard deviation [SD]); weight $18.8 \mathrm{~kg}(+0.2 \mathrm{SD})$; body temperature $38.6^{\circ} \mathrm{C}$; pulse rate, $128 / \mathrm{min}$; respiratory rate $30 / \mathrm{min}$; and blood pressure 89/60 mmHg. There were no abnormal breath sounds and heart murmur. Initial laboratory findings indicated mild inflammation (white blood cell count $12,600 / \mathrm{mm}^{3}$; CRP 3.59 $\mathrm{mg} / \mathrm{dl}$; erythrocyte sedimentation rate 76 $\mathrm{mm} / \mathrm{h}$ ). Chest radiography revealed a normal cardiothoracic ratio (CTR) (48\%) with no consolidation. Electrocardiography revealed a sinus rhythm, a heart rate of 128 beats $/ \mathrm{min}$, and no ST-T change. Echocardiography showed normal left ventricular ejection fraction (70\%) and no coronary dilatation (right coronary artery $2.2 \mathrm{~mm}$; left main coronary artery 2.9 $\mathrm{mm})$.

Clinical course is described in the Figure 1.
Because he had sIgAD, we started oral aspirin $(50 \mathrm{mg} / \mathrm{kg} /$ day) and intravenous urinastatin (50000 U $\times$ 6/day) on day 6; however, his fever persisted. On day 7 , we administered CICsA (3.0 mg/kg/day), and fever subsided soon after 14 hours from CICsA administration (serum concentration was $195 \mathrm{ng} / \mathrm{ml}$ ). On day 12 , WBC and CRP levels improved $\left(8300 / \mathrm{mm}^{3}\right.$ and $0.60 \mathrm{mg} / \mathrm{dl}$, respectively) and CICsA was replaced with oral CsA (3 mg/kg/day). On day 16 , we decreased dosage of aspirin to $5.0 \mathrm{mg} /$ $\mathrm{kg} /$ day and discontinued oral CsA. On day 18, he was discharged without CAL.

\section{Discussion}

This report highlights two important clinical issues. First, CsA is effective for patients with KD without IVIG. Second, sIgAD has a significant role in the pathogenesis of $\mathrm{KD}$.

It is known that IVIG for patients with sIgAD has a high risk of anaphylactic shock ${ }^{1}$. Nishikawa et al. ${ }^{8}$ reported a case of a KD patient associated with sIgAD, who was treated with steroid pulse therapy (intravenous methylprednisolone $30 \mathrm{mg} / \mathrm{kg} /$ day for 3 days) 
and improved without CAL. However, it is well known that steroid therapy for KD without IVIG is a contraindication and increased the incidence of CAL on acute phase of $\mathrm{KD}^{9}$. We started oral aspirin and intravenous urinastatin to avoid anaphylactic shock due to IVIG; however, fever persisted and inflammatory findings did not improve. We previously reported the efficacy of CICsA in patients with IVIG-resistant $\mathrm{KD}$, and the starting dose 3.0 $\mathrm{mg} / \mathrm{kg} /$ day was appropriate with regard to effectiveness and safety ${ }^{3}$. Based on this, we started CICsA instead of IVIG after obtaining informed consent from parents. After 14 hours, he became afebrile and his clinical symptoms improved without CAL. It is possible that the observed efficacy was due to urinastatin; however, fever and inflammatory symptoms were persistent, and he became afebrile and his clinical symptoms improved soon after CICsA. Hence, we concluded that CICsA was effective in this patient. In addition, although the effectiveness of oral CsA for IVIG-resistant $\mathrm{KD}$ is well known, this is the first case of using CsA as a first-line treatment for KD. In addition, CsA is more economical than immunoglobulins or infliximab. Thus, we think that CsA is effective for not only KD patients with sIgAD but also KD patients who cannot use IVIG because of allergy to IVIG or due to religious reasons.

Second, KD associated with sIgAD has important implications in the pathogenesis of KD. Rowley et al. ${ }^{6}$ reported the infiltration of IgA plasma cells in coronary arteries and other inflammatory tissues in acute-phase KD. The etiology of KD is still unknown, and this theory is very interesting. Furthermore, they reported that IgM plasma cells might take over the IgA function in KD patients complicated by $\operatorname{sgAD}{ }^{10}$. We think that IgM plasma cells may partly complement the function of the IgA; the role of the IgA antibody is very important in the inflammation in $\mathrm{KD}$. As a result, the symptoms of KD associated with sIgAD may be milder than those of KD without sIgAD. However, only one KD patient combined with sIgAD has been reported. Further studies are needed to confirm our speculations.

\section{REFERENCES}

1. Yel L. Selective IgA deficiency. J Clin Immunol 2010; 30: 10-16.

2. Kanoh T, Mizumoto T, Yasuda N, et al. Selective IgA deficiency in Japanese blood donors: frequency and statistical analysis. Vox Sang 1986; 50: 81-86.

3. Minami T, Shiraishi $\mathrm{H}$, Oka K, et al. Continuous infusion of cyclosporin $\mathrm{A}$ in intravenous immunoglobulin resistant Kawasaki disease patients. Jichi Medical University Journal 2011; 34: 109-115.

4. Suzuki H, Terai M, Hamada $H$, et al. Cyclosporin A treatment for Kawasaki disease refractory to initial and additional intravenous immunoglobulin. Pediatr Infect Dis J 2011; 30: 871-876.

5. Tremoulet AH, Pancoast P, Franco A, et al. Calcineurin inhibitor treatment of intravenous immunoglobulinresistant Kawasaki disease. J Pediatr 2012; 161: 506512.

6. Rowley AH, Eckerley CA, Jäck HM, et al. IgA plasma cells in vascular tissue of patients with Kawasaki syndrome. J Immunol 1997; 159: 5946-5955.

7. Rowley AH, Shulman ST. Recent advances in the understanding and management of Kawasaki disease. Curr Infect Dis Rep 2010; 12: 96-102.

8. Nishikawa T, Nomura Y, Kono Y, Kawano Y. Selective IgA deficiency complicated by Kawasaki syndrome. Pediatr Int 2008; 50: 816-838.

9. Kato H, Koike S, Yokoyama T. Kawasaki disease: effect of treatment on coronary artery involvement. Pediatrics 1979; 63: 175-179.

10. Rowley AH, Miura M. Immunoglobulin A deficiency and Kawasaki disease. Pediatr Int 2010; 52: 330. 